\title{
A search for LSB dwarf galaxies in the M 81 group on digitally stacked Schmidt plates ${ }^{\star}$
}

\author{
D. Froebrich and H. Meusinger \\ Thüringer Landessternwarte Tautenburg, Sternwarte 5, D-07778 Tautenburg, Germany \\ e-mail: frobrich@tls-tautenburg.de/meus@tls-tautenburg.de
}

Received December 21, 1999; accepted May 18, 2000

\begin{abstract}
We present a search for low surface brightness (LSB) dwarf galaxies in a region of 10 square degrees around M 81. The survey is based on the digital co-addition of Schmidt plates where algorithms for sub-arcsecond co-centering, quality-weighting and sigmaclipping "bad pixel" rejection are applied. The $3 \sigma$ surface brightness limit is $\mu_{\text {lim }}=25.6 \mathrm{mag} / \square^{\prime \prime}$. In a substantial fraction of the surveyed area, the detection of LSB objects is limited by "Galactic cirrus" rather than by the limiting magnitude of the stacked image. Optimum search parameters for an automated detection of dwarf galaxies are derived from simulations. The final selection of LSB dwarf candidates relies on a combination of automated detection and visual inspection. We present six new dwarf galaxy candidates with $\mu_{B}(0) \geq 23 \mathrm{mag} / \square^{\prime \prime}$. Photometric data and best fitting parameters for the exponential model are derived both for the new candidates and for some previously known LSB dwarf galaxies in the field. For the most likely new dwarf member of the M 81 group, a central surface brightness of $\mu_{B}(0)=25.4 \mathrm{mag} / \square^{\prime \prime}$ and a scale-length of $0.3 \mathrm{kpc}$ are derived.
\end{abstract}

Key words: galaxies: clusters: individual: M81 group - galaxies: general — galaxies: photometry — galaxies: fundamental parameters

\section{Introduction}

The M 81 group of galaxies, a condensation in the ComaSculptor cloud (Tully 1987), is one of the nearest groups to our own. The prominent members include the large spiral M 81, the two peculiar galaxies M 82 and NGC 3077, and a

Send offprint requests to: H. Meusinger

* Based on observations made with the $2 \mathrm{~m}$ telescope of the Thüringer Landessternwarte Tautenburg, Germany. few small spirals. The combination of richness, proximity, and high galactic latitude $\left(b \approx 40^{\circ}\right)$ makes this group an ideal probe for its dwarf galaxy population. Moreover, the M 81 group plays an important role in bridging the gap between the investigation of dwarfs in the Local Group and in nearby clusters. Dwarf galaxies are prime targets in the context of such important astronomical issues as cosmic structure formation, primordial chemical abundances, dark matter, galaxy evolution, and star formation.

In an extensive photographic survey, Börngen, Karachentseva, Karachentsev and co-workers have searched for dwarf galaxies in the M 81 group (Börngen \& Karachentseva 1982; Börngen et al. 1982; Börngen et al. 1984; Karachentseva et al. 1984; Karachentseva et al. 1985). The paper by Karachentseva et al. (1985) lists 40 certain, possible or probable members (where membership was found questionable for 14 of them); large scale photographs from the $6 \mathrm{~m}$ telescope are given there for 38 objects. Bremnes et al. (1998; hereafter BBP98) provide CCD photometric data and structure parameters for several dwarfs from this list. Searches for HI emission from optically detected M81 dwarf galaxy candidates have been performed by van Driel et al. (1998) and Huchtmeier \& Skillman (1998). Miller \& Hodge (1994) investigated HII regions of five members of the M 81 group to derive their present star formation rates.

The photographic search for dwarfs in the M 81 group was essentially based on simply surveying Tautenburg Schmidt plates by eye. Despite the success of this method, one may doubt the completeness at low surface brightness. For a more complete survey, the use of large-scale CCDs, more sensitive photographic emulsions, or the method of co-addition of Schmidt plates seems more promising.

A CCD survey over 40 square degrees of the M 81 region has been performed by Caldwell et al. (1998) with the Burrell Schmidt telescope to search for analogs of Local Group dE's. Caldwell et al. note that their survey goes 
probably deeper than the photographic search, but they do not provide accurate survey limits. They recovered all previously known dwarfs in their survey field, along with several new candidates. However, data are given only for one of their candidates (F8D1 in their notation), which is discussed there in detail. Karachentseva \& Karachentsev (1998) made an attempt to increase the completeness of the sample of nearby galaxies by the use of the POSS-II and ESO/SERC plates. Their list of new dwarf galaxy candidates includes 6 further probable members of the M 81 group.

The co-addition (stacking) of several Schmidt plates of the same field provides an interesting alternative for a new, more sensitive photographic survey. Stacking of Schmidt plates is known to be a powerful method for the search of LSB objects in wide fields (e.g., Schweitzer \& Seitzer 1988; Hawkins 1991; Malin \& Carter 1993; Kemp \& Meaburn 1995; Knox et al. 1998; Brunzendorf \& Meusinger 1999a; Meusinger et al. 1999).

The present paper is concerned with the search for LSB dwarf galaxies based on a deep image obtained from the quality-weighted digital stacking of a large number of Tautenburg Schmidt plates. The survey field is centered on M81, the size of the field amounts to 10 square degrees. The M 81 group is, of course, much more extended (see e.g., van Driel et al. 1998, their Fig. 1); the precise extent is not well determined (de Vaucouleurs 1975).

In the following section, we describe the observational material. The plate stacking is the subject of Sect. 3 . Section 4 is concerned with the search procedure. Images and surface brightness profiles for the new candidates and for previously known LSB dwarfs in the field are presented in Sect. 5. Conclusions are given in Sect. 6 .

Throughout this paper, we assume a distance of $3.6 \mathrm{Mpc}$ for the M81 group (Madore et al. 1993), implying a linear scale of $1.05 \mathrm{kpc} \operatorname{arcmin}^{-1}$.

\section{Observational material}

\subsection{Schmidt plates}

The basic observational material of the present study consists of 35 photographic plates from the Tautenburg $2 \mathrm{~m}$ telescope in its Schmidt mode. Each plate covers an unvignetted area of $3.3 \times 3.3$, centered on $\alpha=9^{\mathrm{h}} 55 \mathrm{~m}^{\mathrm{m}} 6, \delta=68^{\circ} 04^{\prime}(\mathrm{J} 2000)$. The plate scale amounts to $51^{\prime \prime} .4 \mathrm{~mm}^{-1}$. 27 plates were selected from the Tautenburg plate archive, further 8 plates were taken in 1997 and 1998 in preparation of the present project. For the majority (30) of the plates nearly identical emulsions were used. These emulsions, in combination with a Schott GG 13 filter, closely match the standard $B$ band. Three plates have blue-sensitive emulsions but were taken either without filter or through a Schott GG 11 filter. Two other plates have a Kodak IIIa-J emulsion in combination with a GG 13 filter. 31 plates have a calibration wedge exposed onto.

\section{2. $C C D$ observations}

CCD frames in the $R$ band and in a $100 \AA$ wide band around $\mathrm{H} \alpha$ were taken for some sub-areas of the field. The Tautenburg Schmidt telescope was used, equipped with a SITe chip of $2128 \times 2128$ pixels with pixel size of $24 \mu \mathrm{m} \times 24 \mu \mathrm{m}$. Total exposure times were about $1000 \mathrm{~s}$ to $2000 \mathrm{~s}$ in $R$ and $2000 \mathrm{~s}$ in $\mathrm{H} \alpha$. The weather conditions were in general good, though not always photometric. The seeing was $2 \ldots 3^{\prime \prime}$, comparable with the mean seeing on the photographic plates. The image reduction was done with the IRAF package. The zero-point of the $R$ band magnitude scale was calibrated with the magnitude scale given by Zickgraf et al. (1990).

\section{Plate stacking procedure}

All plates were digitised by means of the Tautenburg Plate Scanner (Brunzendorf \& Meusinger 1999a, 1999b) with a resolution of $10 \mu \mathrm{m}$ (corresponding to $0^{\prime \prime} .5$ ). The procedure applied for stacking digitised wide-field plates includes the following steps: (a) conversion of the measured photographic transparencies into relative intensities, (b) computation of weighting factors to account for the variation of the quality from plate to plate, (c) subdivision of the plate and accurate adjustment of the subframes, (d) removal of spurious information (like satellite trails, scratches, or emulsion flaws), (e) weighted pixel-by-pixel addition of the subframes, and (f) photometric calibration of the resulting image.

To compute relative intensities from measured transparencies, an analytical transformation relation (Lehmann \& Häupl 1987) was used with parameters derived by individual least-square fits on the corresponding calibration wedges. An average wedge was adopted for the few plates where no wedges were exposed onto.

The quality of the plates, e.g. in terms of limiting magnitudes, shows a considerable scatter. Therefore, the coaddition should consider weighting factors in order to yield an optimal signal-to-noise ratio (Richter 1978; Knox et al. 1998). The weighted co-addition of $N$ plates with individual signal-to-noise ratios $S_{i} / \sigma_{i}(i=1 \ldots N)$ and weighting factors $\omega_{i}$ leads to a resulting sinal-to-noise ratio of

$$
\frac{S_{\Sigma}}{\sigma_{\Sigma}}=\frac{S_{1}+\sum_{i=2}^{N} \omega_{i} S_{i}}{\sqrt{\sigma_{1}^{2}+\sum_{i=2}^{N} \omega_{i}^{2} \sigma_{i}^{2}}}
$$



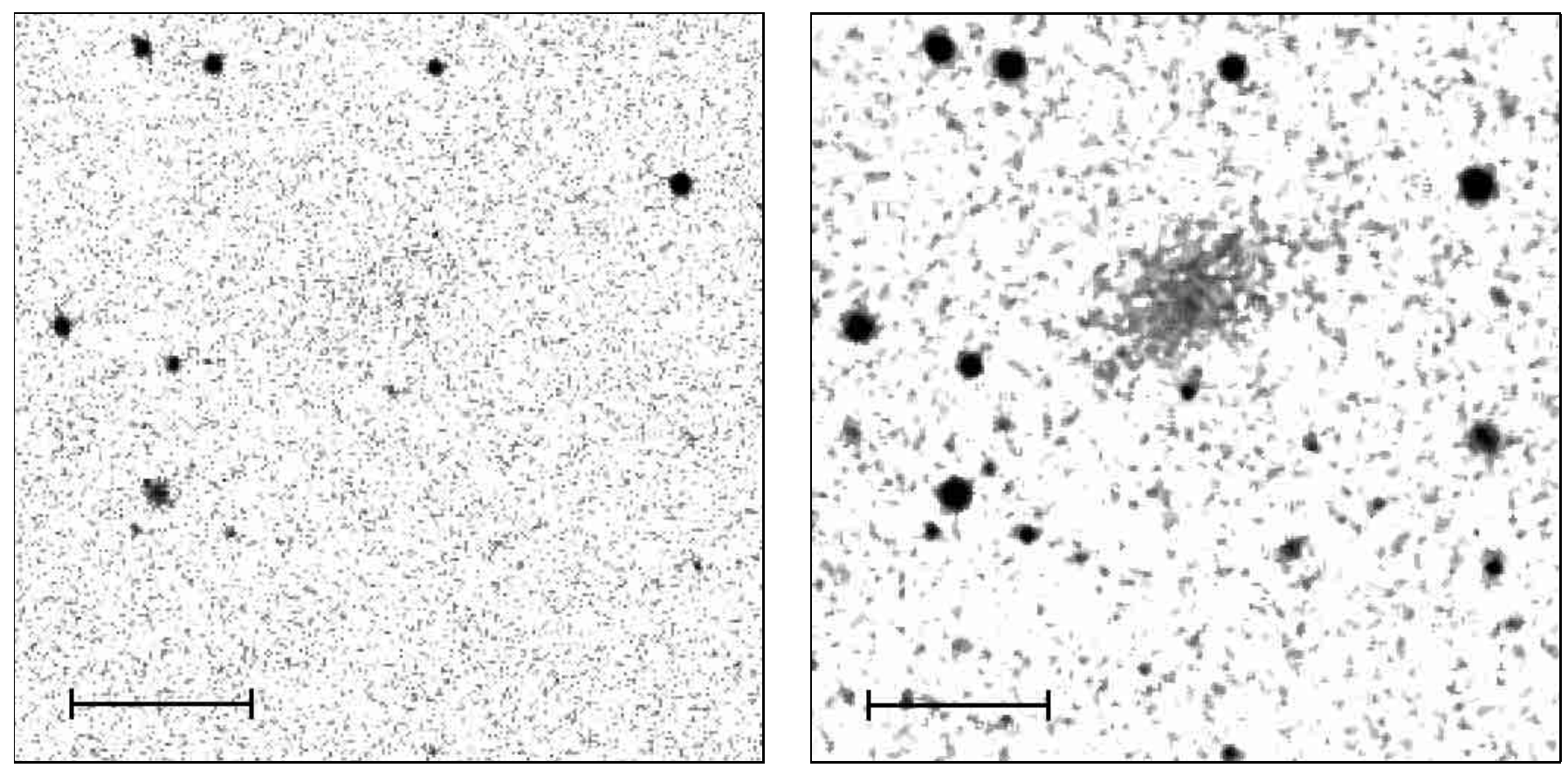

Fig. 1. The LSB dwarf galaxy BK 5N: comparison of the image from the master-plate (left) and from the quality-weighted stacked image slightly smoothed with a median filter (right). The horizontal bar indicates $1^{\prime}$

where the index 1 denotes the master-plate with a weighting factor $\omega_{1}=1$. The maximum signal-to-noise ratio is attained for weighting factors

$\omega_{i}=\frac{S_{i} \sigma_{1}^{2}}{S_{1} \sigma_{i}^{2}}$

We computed the weighting factors according to Eq. (2) for 30 faint objects which are detectable on all plates. The mean value of the $\omega_{i, k}(k=1 \ldots 30)$ measured on a given plate $i$ was taken as the plate weighting factor of this plate. The gain in limiting magnitude due to the weighted co-addition is given by

$G=2.5 \log _{10} \sqrt{1+\sum_{i=2}^{N}\left(\frac{S_{i} / \sigma_{i}}{S_{1} / \sigma_{1}}\right)^{2}}$.

Stacking of the plates without quality-weighting leads to a gain in limiting magnitude of $1.4 \mathrm{mag}$ relative to the master-plate. The consideration of weighting factors yields a gain of $1.8 \mathrm{mag}$ (Fig. 1).

All digitised plates were sub-divided into 25 equalsized areas of $5400 \times 5566$ pixels. In subimages of this size, differential refraction makes an effect of only 0.5 and can be ignored. The positional accuracy of the co-addition is estimated to be better than 0.5 , i.e. less than the pixelsize. This means that the spatial resolution is not substantially affected by the stacking procedure.

Prior to the co-addition of the subimages, a sigmaclipping algorithm for the rejection of spurious informations was applied. Even though great care was taken on the removal of false structures, it can never be completely excluded that a faint structure detected on a single stacked image is a remnant of incomplete "bad pixel" treatment. Therefore, we decided to create two further stacks, in addition to the stack from all plates, which are used to verify faint structures detected on the search stack image. These two verification stacks have nearly identical limiting magnitudes and are independent from each other in the sense that there was no plate included in both of these stacks. A faint structure is considered as relevant if it is detected not only on the search stack but on both of the verification stacks as well.

To determine photometric properties of the galaxies, a fourth stack was created including only the 31 plates with a calibration wedge exposed on. (In this way, four of the five plates which deviate from the standard $B$ band were omitted.) For the external calibration of the relative intensities, photometric standard stars from Zickgraf et al. (1990) were used which have typical uncertainties of 0.04 mag. After the subtraction of the mean offset between the magnitudes from Zickgraf et al. and our measured internal magnitudes, the remaining rms difference between our data and those from the literature is $0.03 \mathrm{mag}$ (Fig. 2).

To summarize, altogether four stacks of plates were used in the present study: a stack of all plates to search for LSB objects, two independent stacks for checking discovered objects, and a further stack for photometry. The photometric stack reaches a $3 \sigma$ surface brightness limit of $25.6 \pm 0.1 \mathrm{mag} / \square^{\prime \prime}$, corresponding to $2.4 \%$ of the sky background.

It is known for a long time that much of the field around M 81 is covered with faint, filamentary nebulosities due to "Galactic cirrus" (Sandage 1976; Appleton et al. 


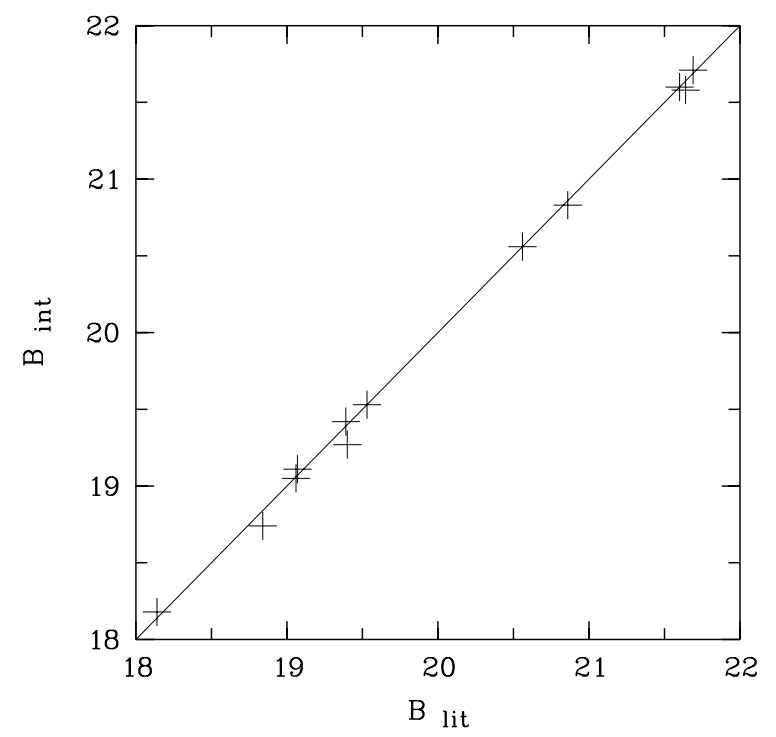

Fig. 2. Photometric calibration. The magnitudes of the calibration stars (crosses) were taken from Zickgraf et al. (1990)

1993). The $V$ surface brightness of the most prominent filamentary nebulosities in the area is $\mu_{V} \approx 25 \mathrm{mag} / \square^{\prime \prime}$ (Sandage 1976). Despite the fact that the brightest nebulosities shown by Sandage (1976) are outside of our survey field, the "cirrus" affects the detection of LSB objects in a significant fraction of the region of the present study.

\section{Search for LSB dwarf galaxy candidates}

\subsection{Simulations of the appearance of $L S B$ dwarf galaxies}

Before starting a search for dwarf galaxy candidates of the M 81 group, it is instructive to get an impression of the expected appearance of such objects on our stacked images. Therefore, we performed simulations of dwarf galaxies with properties taken from the list of Local Group dwarfs (Mateo 1998) but with distances corresponding to the M81 group. We restricted the simulations to dwarf spheroidal galaxies because these are the most frequent LSB systems. Moreover, it is well known that the M 81 group, like other groups and clusters, shows a morphological segregation in the sense that early-type dwarfs are more concentrated in the central region than late-type dwarfs (e.g., van Driel et al. 1998).

The radial intensity profiles of dwarf galaxies are well described by an exponential law (e.g., Binggeli \& Cameron 1993) with two parameters: the central surface brightness $\mu(0)$ and the scale-length $l$. We simulated such profiles where $\mu_{B}(0)$ was varied from $24.5 \mathrm{mag} / \square^{\prime \prime}$ down to $26.5 \mathrm{mag} / \square^{\prime \prime}$ in steps of $0.5 \mathrm{mag} / \square^{\prime \prime}$ and $l$ from $0.1 \mathrm{kpc}$ to $0.5 \mathrm{kpc}$ in steps of $0.1 \mathrm{kpc}$. The simulated galaxies were projected onto various background images selected by chance from the stacked image of the M 81 field. The simulation of every galaxy type (i.e., a combination of $\mu_{B}(0)$ and $l$ ) was repeated 50 times with different backgrounds (i.e., surface brightness and environment) for each simulation. Figure 3 shows a part of the simulation mosaic.

Next, a median filter was applied to each simulated image to improve the detectibility of LSB objects due to an enhancement of the signal-to-noise ratio (Irvin et al. 1989). The radius of the filter was varied between $r_{\mathrm{f}}=1^{\prime \prime}$ and $5 "$. Finally, the detection rate of the simulated galaxies was determined in dependence on $\mu_{B}(0), l$ and $r_{\mathrm{f}}$. The conclusions from the simulations are: first, the detection rate is significantly enhanced in the filtered images compared to the unfiltered ones for $\mu_{B}(0)>25 \mathrm{mag} / \square^{\prime \prime}$. The highest detection rate is achieved for $r_{\mathrm{f}}=2^{\prime \prime}$, independent of the galaxy parameters. Therefore, we have processed the images with a median filter of $r_{\mathrm{f}}=2^{\prime \prime}$ prior to the search for LSB dwarfs. Second, the simulations allow an estimation of the success rate (i.e., the detection probability) of the search. For $\mu_{B}(0) \leq 25 \mathrm{mag} / \square^{\prime \prime}$, a success rate of 90 per cent is expected. For $\mu_{B}(0)=25$ to $26 \mathrm{mag} / \square^{\prime \prime}$, the expected value is $80 \ldots 90$ per cent, and for $\mu_{B}(0)=26.5 \mathrm{mag} / \square^{\prime \prime}$ it is still between 50 and 75 per cent.

\subsection{Search procedure}

The median-filtered stacked image was processed with a modified version of the object detection software from the Münster Redshift Project (Horstmann et al. 1989). In a next step, the detected objects had to be classified to discriminate extended objects against star-like objects. The selection parameter is defined following Maddox et al. (1990) and is determined in an iteration process in two steps: at first, we determined the deviation of the measured profile from the Gaussian for the same magnitude by the profile residuum

$\Phi^{(\mathrm{G})}(m)=\log _{10} \sum_{i=1}^{8}\left[r_{i}(m)-r_{i}^{(\mathrm{G})}(m)\right]^{2}$,

where $r_{i}(m)$ is the radius of the image at the $i$ th intensity level, $r_{i}^{(\mathrm{G})}(m)$ is the width of the Gaussian profile at level $i$.

As the intensity profile of a star-like object is well described by a two-dimensional Gaussian, the sequence of star-like objects is clearly separated from the resolved objects in the $\Phi^{(\mathrm{G})}$ versus $m$ diagram. In this way, a stellar sequence is defined which is used, in a second step, to derive a refined profile residuum, $\Phi^{*}$, which measures directly the deviation of the measured profile from the stellar profile of the same magnitude:

$\Phi^{*}(m)=\log _{10} \sum_{i=1}^{8} \frac{1}{\sigma_{i}^{2}(m)}\left[r_{i}(m)-\overline{r_{i}(m)}\right]^{2}$, 


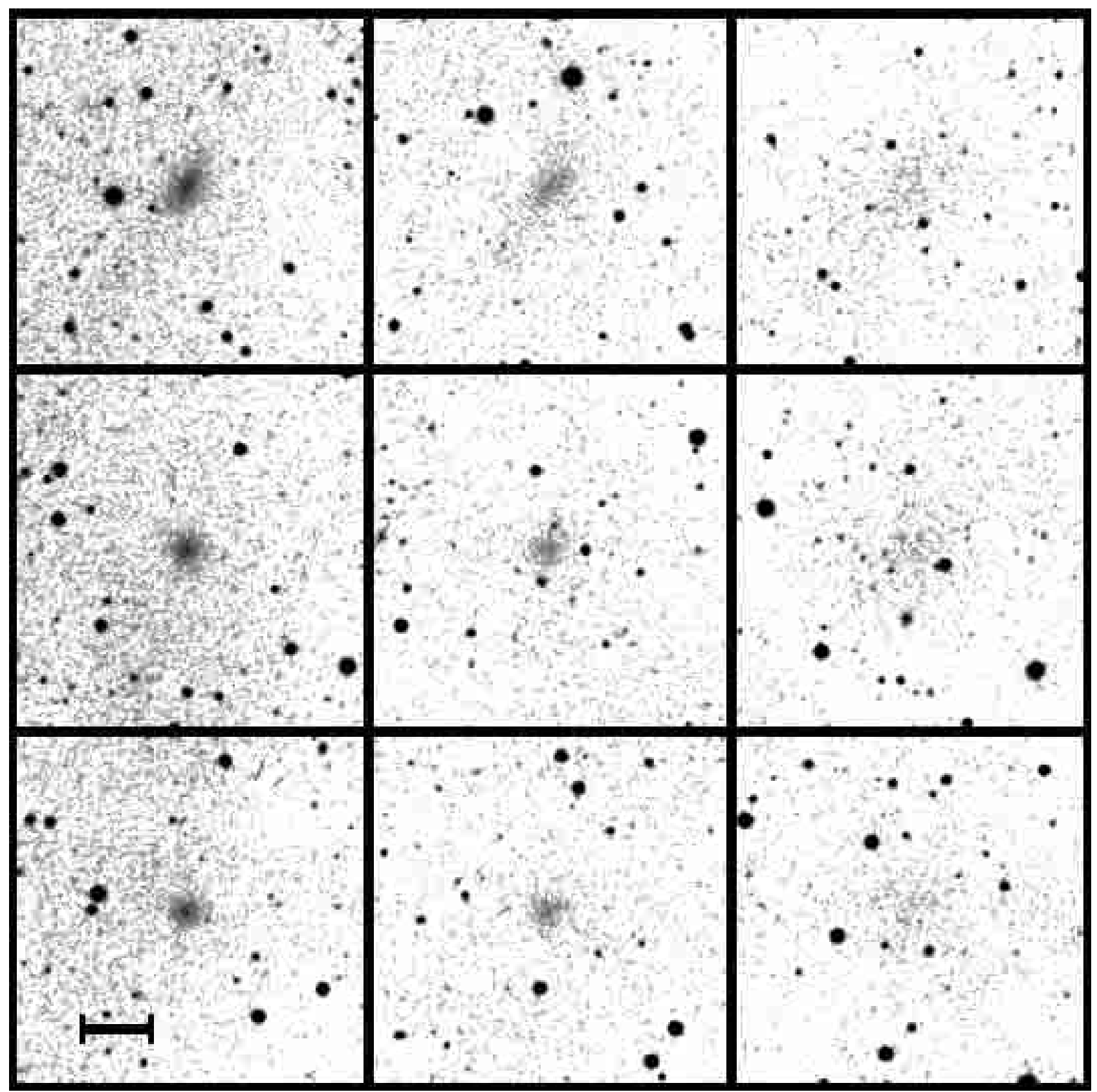

Fig. 3. Mosaic of nine examples of simulated dwarf galaxies at the distance of the $\mathrm{M} 81$ group. The central surface brightness of the galaxies is $24.5 \mathrm{mag} / \square^{\prime \prime}$ (left), $25.5 \mathrm{mag} / \square^{\prime \prime}$ (middle), and $26.5 \mathrm{mag} / \square^{\prime \prime}$ (right). Different axis ratios were simulated by the assumption of a disk-like structure with different inclinations (from bottom to top: $0^{\circ}, 30^{\circ}, 60^{\circ}$ ). A scale-length of $0.3 \mathrm{kpc}$ was adopted for the galaxies in this simulation. The length of the horizontal bar in the lower left panel corresponds to one arcminute

where $\overline{r_{i}(m)}$ is the mean radius of the images of the starlike objects with the apparent magnitude $m$ at intensity level $i$, and $\sigma_{i}(m)$ is the standard deviation of the $r_{i}(m)$. There is a clear separation between star-like objects and resolved objects, at least for $B<20.5$ (Fig. 4). We consider all objects with $\Phi^{*} \leq 1.5$ as star-like. The sample of objects with $\Phi^{*}>1.5$ essentially contains a mixture of galaxies and double objects.
Since the galaxy selection by the automated procedure is not unambiguous, all objects classified as nonstellar were inspected by eye. In addition, the whole stacked plate was inspected by eye for galaxies probably not detected by the automated procedure. We selected all galaxies with a central surface brightness $\mu_{B}(0) \geq 23 \mathrm{mag} / \square^{\prime \prime}$ and a diameter greater then $8^{\prime \prime}$ as candidates for LSB dwarf galaxy members of the $M$ 81-group. The search result was 


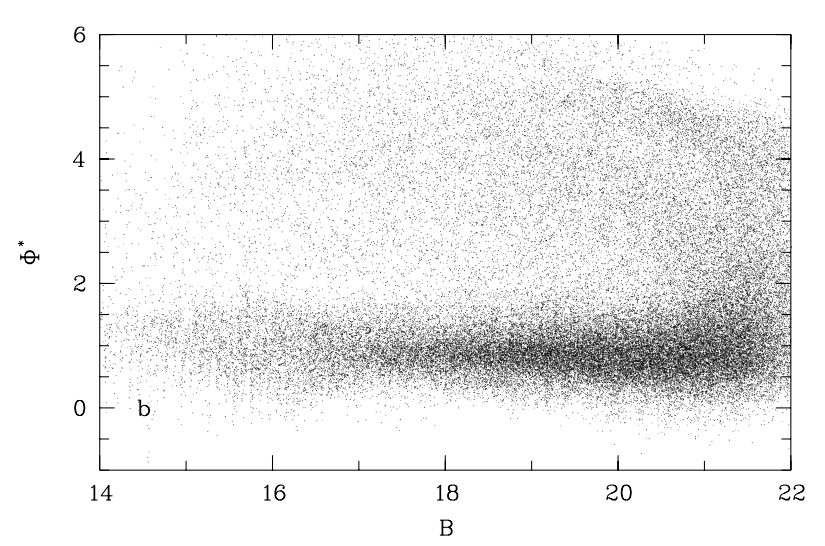

Fig. 4. Star-galaxy separation: profile residua $\Phi^{*}$ for all objects detected on the image from the search stack (see text)

Table 1. New LSB dwarf galaxy candidates

\begin{tabular}{lccl}
\hline name & $\begin{array}{c}\text { R.A. (J2000) } \\
(\mathrm{h} \mathrm{m} \mathrm{s})\end{array}$ & $\begin{array}{c}\text { Dec. (J2000) } \\
\left({ }^{\circ}{ }^{\prime}{ }^{\prime \prime}\right)\end{array}$ & type \\
\hline cand1 & 094510.0 & +684554 & $\mathrm{dSph} ?$ \\
cand2 & 095807.0 & +693601 & $\mathrm{dE} ?$ \\
cand3 & 094316.0 & +682444 & $\mathrm{Im} ?$ \\
cand4 & 094755.0 & +675436 & $\mathrm{Im} ?$ \\
cand5 & 093902.0 & +692501 & $\mathrm{Im} ?$ \\
cand6 & 100941.0 & +684712 & $\mathrm{dSph} ?$ \\
\hline
\end{tabular}

compared with the list of known galaxies in the field from the $\mathrm{NED}^{1}$.

Altogether six new possible LSB dwarf galaxies were detected (Table1). In addition, we detected all of the dwarfs members and member candidates previously known in the search field, with the only exception of F8D1 which is located near the edge. (Note that, due to plate-to-plate variations of the centre coordinates, the margins of our survey area are covered by a substantially smaller number of plates and are, therefore, not as deep as the inner part of the field. Note further that F8D1 has an exceptionally low surface brightness; Caldwell et al. 1998.)

\section{Description of the new dwarf galaxy candidates}

\subsection{Photometric parameters}

Photometric parameters were determined by use of the "growth curve", $F(r)$, (see BBP98). The total apparent magnitude $m$ is given by

$m=-2.5 \log _{10} F(\infty)$,

1 The NASA/IPAC Extragalactic Database (NED) is operated by the Jet Propulsion Laboratory, California Institute of Technology, under contract with the National Aeronautics and Space Administration.

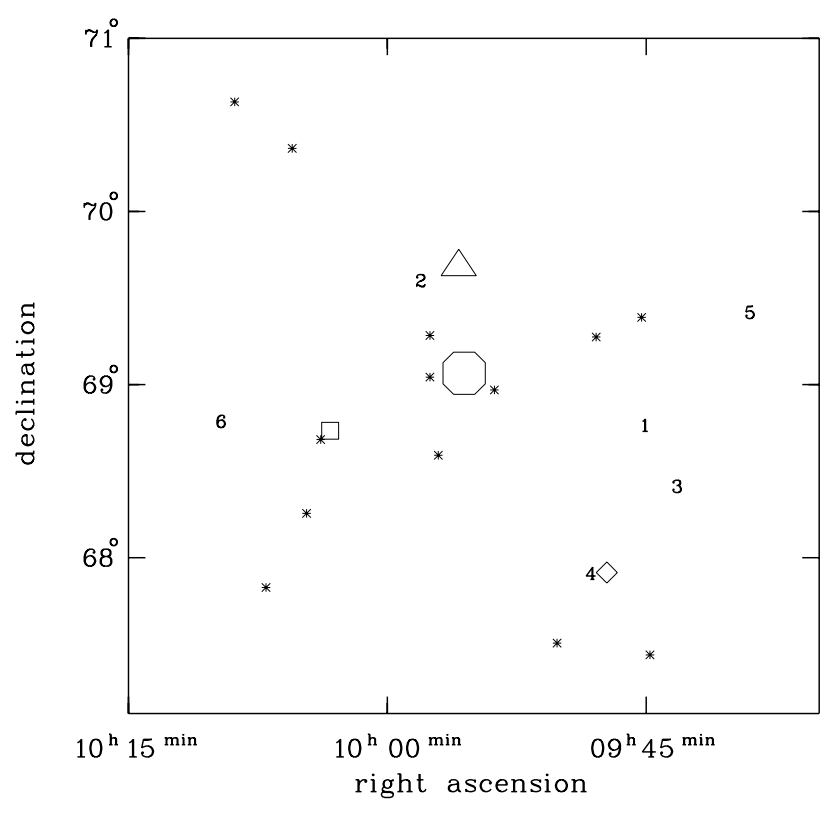

Fig. 5. Sky map of the survey field around M 81. The larger galaxies are indicated by individual symbols (hexagon: M 81, triangle: M 82, square: NGC 3077, lozenge: NGC 2976), the other previously known galaxies are shown as asterisks, and the new objects are indicated by their candidate numbers

and the average surface brightness within the effective radius $r_{\text {eff }}$ is

$<\mu>_{\text {eff }}=m+5 \log _{10}\left(r_{\text {eff }}\right)+2.5 \log _{10}(2 \pi)$,

where $r_{\text {eff }}$ is in arcsec and $\mu$ in mag/ $\square^{\prime \prime}$.

The intensity profiles of dwarf galaxies are well fitted by an exponential law, $I(r)=I_{0} \mathrm{e}^{-\alpha r}$, (Binggeli \& Cameron 1993) with scale-lengths $l=1 / \alpha$. In terms of the surface brightness, the exponential model is expressed by

$\mu^{\mathrm{ex}}(r)=\mu^{\mathrm{ex}}(0)+1.086 \alpha r$,

where $\mu(0)^{\mathrm{ex}}$ is the surface brightness extrapolated towards the centre. The upper index "ex" refers to the exponential fit. According to the exponential model, the apparent magnitude is given by

$m^{\mathrm{ex}}=\mu^{\mathrm{ex}}(0)+5 \log _{10} \alpha-2.5 \log _{10}(2 \pi)$,

and the corresponding colour gradient is

$\left[\frac{\mathrm{d}(B-R)}{\mathrm{d} r}\right]^{\mathrm{ex}}=1.086\left(\alpha_{B}-\alpha_{R}\right)$.

Before estimating photometric parameters, the images were corrected for the sky background, the contamination by foreground stars, and Galactic absorption adopting $A_{B}=0.08 \mathrm{mag}$ and $A_{R}=0.05 \mathrm{mag}$ (BBP98).

The results are listed in Tables 2 and 3 for the six new candidates and for some of the known galaxies. The systems F8D1, UGC 5423, and UGC 5455 were not considered here because they are located close to the edge of our survey field, where the background is quite inhomogenous. 

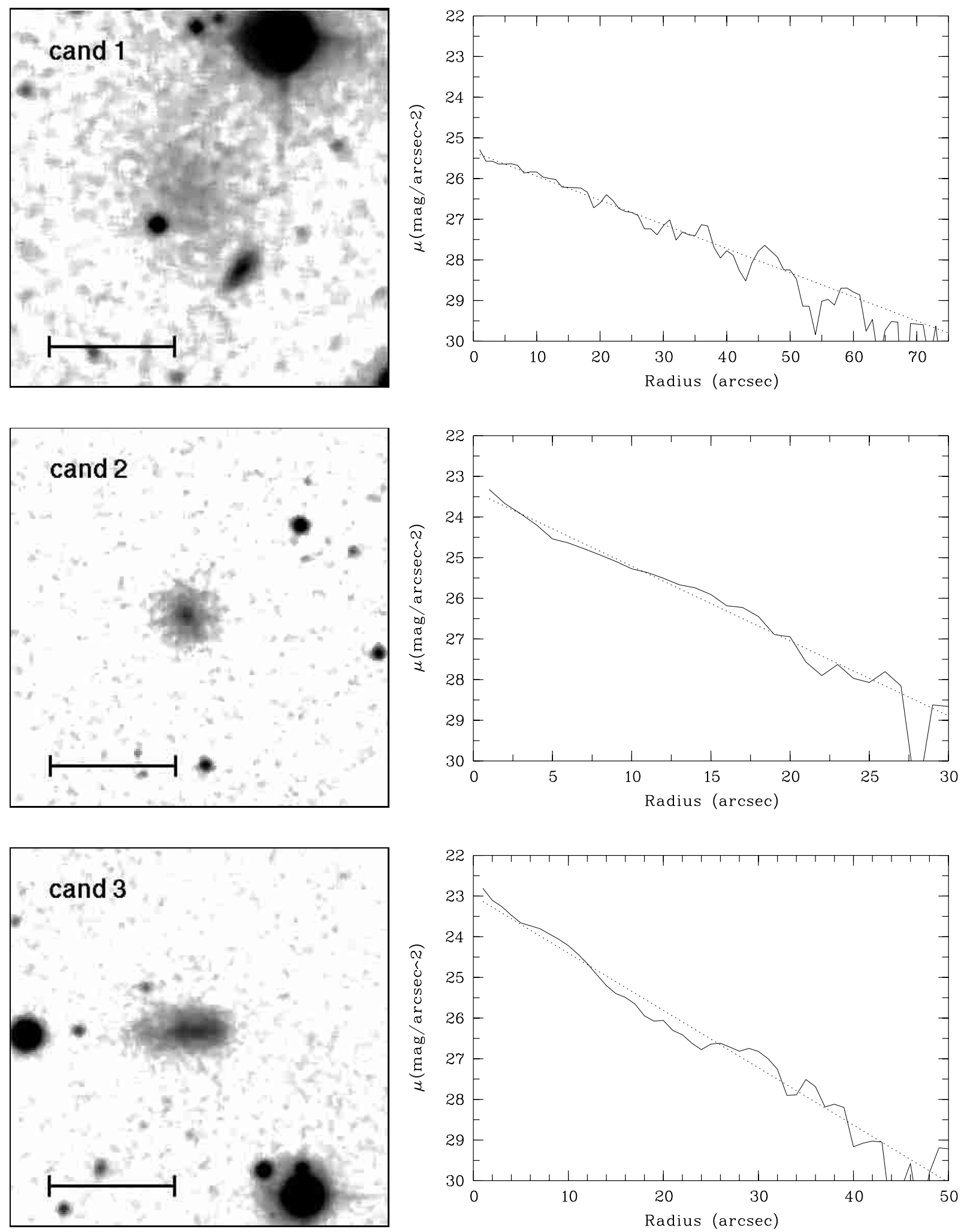

Fig. 6. Images and $B$ surface brightness profiles for the dwarf galaxy candidates 1,2 , and 3 . The dotted lines represent the exponential fits. The horizontal lines indicate $1^{\prime}$ 

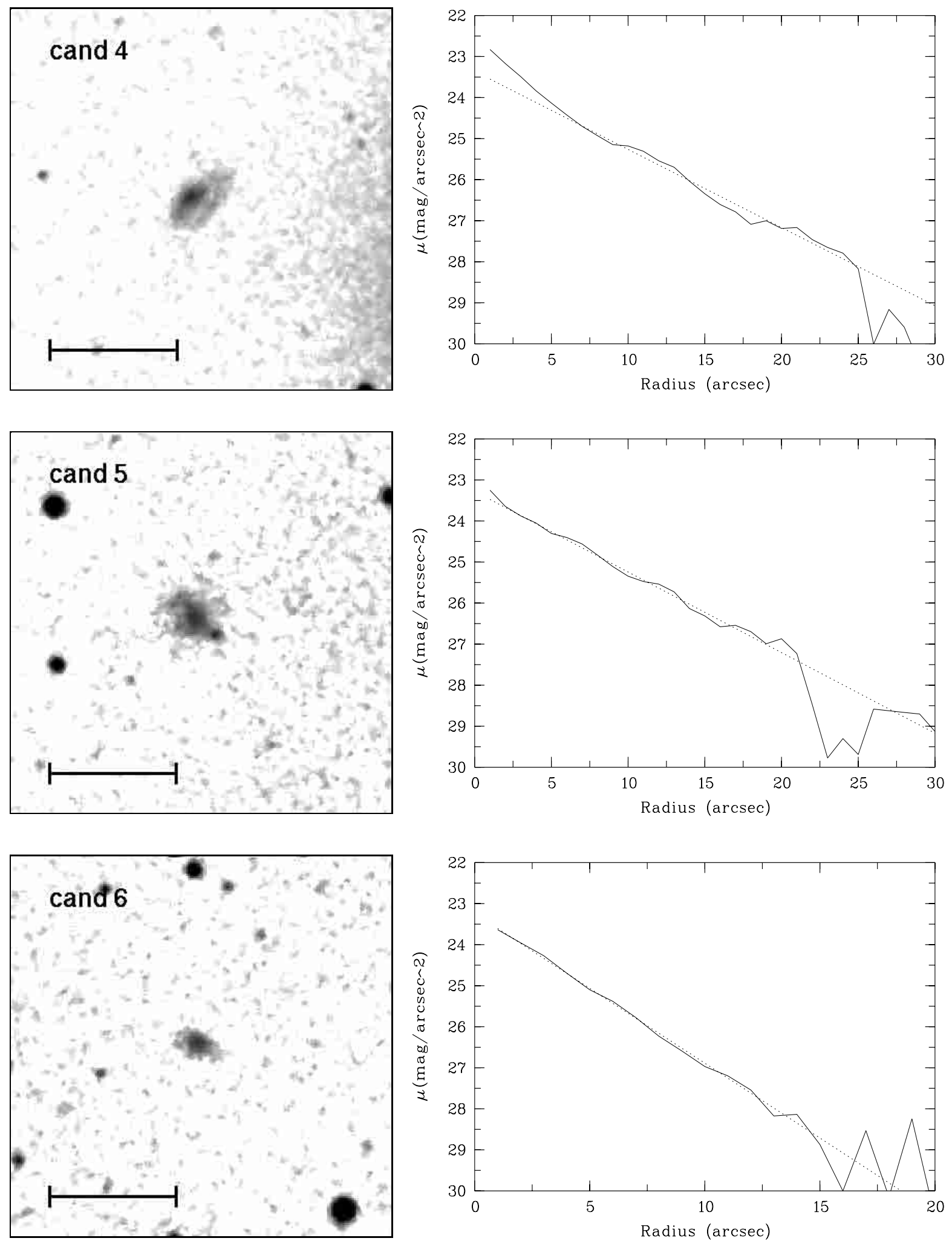

Fig. 7. As Fig. 6, but for the dwarf galaxy candidates 4, 5, and 6 

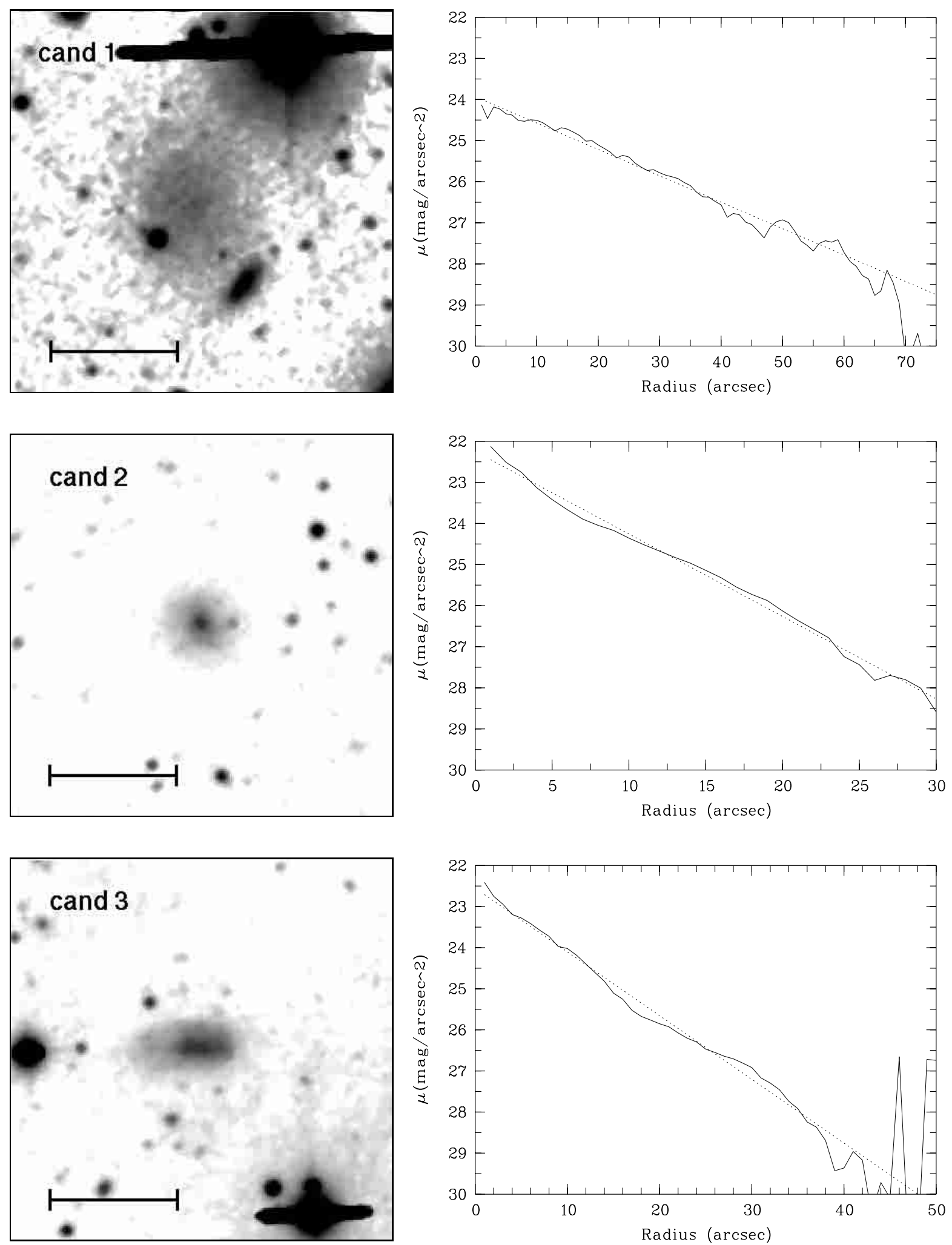

Fig. 8. As Fig. 6 , but for the $R$ band 
Table 2. Global photometric properties in $B$ and $R$ for the six new LSB dwarf galaxy candidates and for known LSB dwarfs in the suvey field. (The radii $r$ are given in arcsec, where the numbers in the index refer to the corresponding isophote in mag/ $\square^{\prime \prime}$ )

\begin{tabular}{|c|c|c|c|c|c|c|c|c|c|c|c|c|}
\hline name & $B$ & $B-R$ & $r_{B, \text { eff }}$ & $r_{R, \mathrm{eff}}$ & $\left\langle\mu_{B}\right\rangle_{\text {eff }}$ & $\left\langle\mu_{R}\right\rangle_{\mathrm{eff}}$ & $r_{B, 25}$ & $r_{B, 26}$ & $r_{B, 27}$ & $r_{R, 25}$ & $r_{R, 26}$ & $r_{R, 27}$ \\
\hline cand1 & 17.2 & 1.3 & 26.3 & 24.9 & 26.3 & 24.9 & - & 10.2 & 25.9 & 17.4 & 32.9 & 45.9 \\
\hline cand2 & 17.6 & 1.0 & 10.4 & 9.7 & 24.7 & 23.5 & 8.3 & 15.3 & 19.9 & 14.0 & 19.6 & 23.8 \\
\hline cand3 & 16.6 & 0.2 & 11.6 & 11.3 & 23.9 & 23.6 & 13.2 & 19.1 & 28.7 & 14.8 & 21.4 & 30.3 \\
\hline cand4 & 17.4 & - & 8.5 & - & 24.1 & - & 8.1 & 13.7 & 18.2 & - & - & - \\
\hline cand5 & 17.6 & - & 8.7 & - & 24.3 & - & 8.5 & 13.5 & 19.9 & - & - & - \\
\hline cand 6 & 18.8 & - & 5.2 & - & 24.4 & - & 4.7 & 7.3 & 10.0 & - & - & - \\
\hline BK 1N & 16.9 & - & 17.3 & - & 25.1 & - & 9.8 & $\overline{19.6}$ & 25.5 & - & - & - \\
\hline $\mathrm{BK} 2 \mathrm{~N}$ & 18.0 & 0.9 & 12.6 & 11.0 & 25.5 & 24.3 & 4.9 & 9.9 & 17.1 & 10.7 & 17.6 & 23.6 \\
\hline $\mathrm{BK} 3 \mathrm{~N}$ & 18.6 & 0.5 & 8.0 & 9.2 & 25.1 & 25.0 & 3.6 & 9.6 & 11.4 & 6.9 & 10.0 & 14.5 \\
\hline BK $5 \mathrm{~N}$ & 17.5 & - & 19.6 & - & 26.0 & - & - & 11.6 & 25.3 & - & - & - \\
\hline KAR 61 & 15.3 & 1.5 & 47.8 & 61.6 & 25.7 & 24.8 & 11.4 & 35.1 & 64.2 & 39.5 & 79.8 & 116.1 \\
\hline KAR 64 & 15.4 & - & 38.5 & - & 25.3 & - & 16.3 & 34.0 & 54.3 & - & - & - \\
\hline KAR 96 & 15.8 & - & 46.9 & - & 26.2 & - & - & 18.5 & 58.6 & - & - & - \\
\hline
\end{tabular}

Table 3. Parameters from the fit of the exponential model for the objects from Table 2 . The upper index ex refers to the exponential model, $l_{B}$ and $l_{R}$ are the scale-lengths of the $B$ and $R$ images in kpc

\begin{tabular}{lccccccccc}
\hline name & $B^{\mathrm{ex}}$ & $\mu_{B}^{\mathrm{ex}}(0)$ & $\mu_{R}^{\mathrm{ex}}(0)$ & $l_{B}$ & $l_{R}$ & $B^{\mathrm{ex}}-B$ & $R^{\mathrm{ex}}-R$ & {$\left[\frac{\mathrm{d}(B-R)}{\mathrm{d} r}\right]^{\mathrm{ex}}$} & $B-R^{\mathrm{ex}}$ \\
\hline cand1 & 17.0 & 25.4 & 23.9 & 0.32 & 0.30 & -0.19 & -0.08 & -0.005 & 1.2 \\
cand2 & 17.5 & 23.4 & 22.3 & 0.10 & 0.09 & -0.06 & -0.02 & -0.017 & 0.9 \\
cand3 & 16.6 & 23.0 & 22.6 & 0.13 & 0.12 & -0.03 & -0.03 & -0.014 & 0.3 \\
cand4 & 17.6 & 23.4 & - & 0.12 & - & +0.15 & - & - & - \\
cand5 & 17.6 & 23.3 & - & 0.09 & - & -0.01 & - & - & - \\
cand6 & 18.9 & 23.2 & - & 0.05 & - & +0.07 & - & - & - \\
\hline BK 1N & 16.8 & 24.2 & - & 0.20 & - & -0.03 & - & - & - \\
BK 2N & 17.9 & 24.4 & 23.1 & 0.13 & 0.11 & -0.06 & -0.07 & -0.028 & 0.9 \\
BK 3N & - & - & - & - & - & - & - & - & - \\
BK 5N & 17.5 & 24.8 & - & 0.20 & - & +0.02 & - & - & - \\
KAR 61 & 15.2 & 24.5 & 23.7 & 0.50 & 0.67 & -0.08 & -0.02 & +0.008 & 1.4 \\
KAR 64 & 15.6 & 24.0 & - & 0.34 & - & +0.18 & - & - & - \\
KAR 96 & 15.3 & 25.4 & - & 0.74 & - & -0.53 & - & - & - \\
\hline
\end{tabular}

The systems Ho IX, A952+69, and the "garland" were not analysed because of their irregular structure which is not well fitted by the exponential model.

The style of Table 2 was adopted from BBP98. Thus, our results of the photographic photometry can be directly compared with their data from CCD photometry. For the 6 dwarf galaxies BK $1 \mathrm{~N}, \mathrm{BK} 2 \mathrm{~N}, \mathrm{BK} 3 \mathrm{~N}, \mathrm{BK} 5 \mathrm{~N}$, Kar 61, and Kar 64 contained both in our survey and in the list of BBP98, we find the following mean differences (here - BBP98): $\langle\Delta B\rangle=-0.13 \pm 0.23,\left\langle\Delta\left\langle\mu_{B}\right\rangle_{\text {eff }}\right\rangle=$ $-0.11 \pm 0.20 \mathrm{mag} / \square^{\prime \prime},\left\langle\Delta \mu_{B}^{\mathrm{ex}}(0)\right\rangle=-0.16 \pm 0.27 \mathrm{mag} /$ $\square^{\prime \prime},\left\langle\Delta r_{27} / r_{27}\right\rangle=-0.06 \pm 0.05,\left\langle\Delta l_{B} / l_{B}\right\rangle=0.11 \pm 0.21$. For comparison, BBP98 estimate a total magnitude error of the order of $0.2 \mathrm{mag}$ due to uncertainties of the zero-point determination and of the correction for sky backround. A similar error is expected for our results. Moreover, there is a further source of uncertainties in our data due to the uncertainty of the extrapolation of the $B$ band calibration curve towards faintest magnitudes. With respect to these error estimates, the agreement between our results and those from BBP98 is remarkably good.

\subsection{Images and radial surface brightness profiles}

In Figs. 6 and 7, we present $B$ images of the six new dwarf galaxy candidates along with the corresponding radial surface brightness profiles and the exponential fits. CCD images in the $R$ band and in $\mathrm{H} \alpha+[\mathrm{N}$ II] are available for the dwarf candidates 1,2 , and 3 . The remaining three candidates were discovered, unfortunately, after the CCD observation campaigns. The $R$ images are shown in Fig. 8, again in combination with the surface brightness profiles. The $(B-R)$ colour profiles are shown in Fig. 11 .

The $\mathrm{H} \alpha$ images were carefully corrected for continuum emission by subtracting the $R$ band images normalized in such a way that most of the stars disappear on the difference images. There is no indication for significant $\mathrm{H} \alpha$ emission found for the three galaxies. Therefore, the $\mathrm{H} \alpha$ images are not shown. 

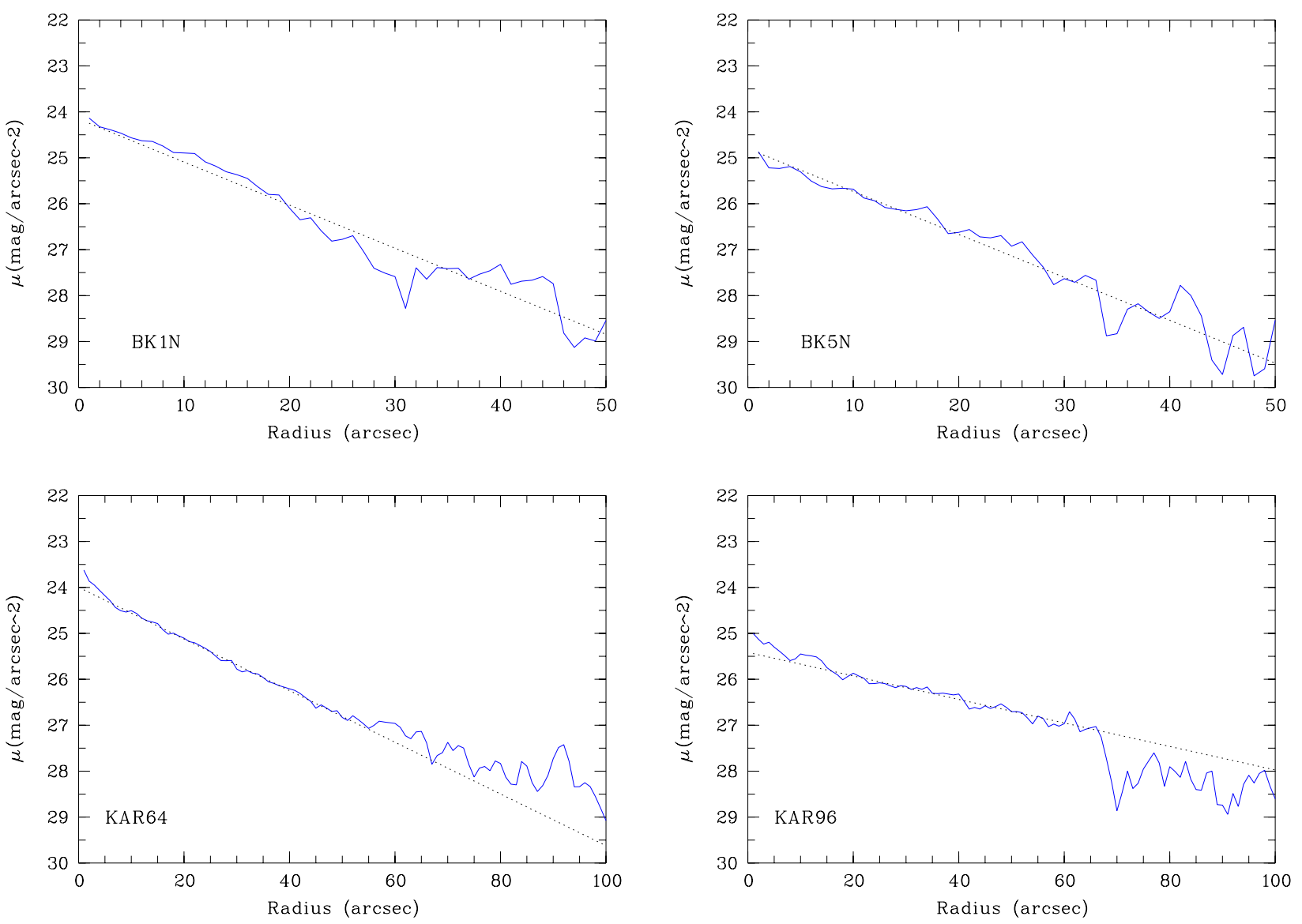

Fig. 9. $B$ band surface brightness profiles for the dwarf galaxies BK 1N, BK 5N, KAR 64, and KAR 96. The dotted lines represent the exponential fits

In Figs. 9 and 10, we present also radial $B$ surface brightness profiles for 7 previously known galaxies. (For images of these systems, see BBP98.) Three of these galaxies were also observed in $R$ (Fig. 10). In general, there is good agreement with the profiles shown by BBP98. Note also, that the exponential model provides a good fit to the radial profiles, with the only exception of BK $3 \mathrm{~N}$. Colour profiles for three of these galaxies are given in Fig. 11.

\subsection{Individual descriptions of the new LSB dwarf galaxy candidates}

Cand 1 is perhaps the candidate with the highest M 81 group membership probability. Moreover, if it belongs to the group, it is one of the members with the lowest surface brightness known so far (a lower surface brightness was measured only for F8D1, Caldwell et al. 1998). Cand 1 appears rather structureless. Unfortunately, the image analysis is handicapped by both a bright foreground star close to and a fainter foreground star projected onto the galaxy. The global properties of cand 1 are reminiscent of BK $5 \mathrm{~N}$, but are in fact between BK 5N and F8D1. The radial surface brightness profiles are well fitted by the exponential model with a relative large scale-length of about $0.3 \mathrm{kpc}$ and without any indication for a steepening of the profile towards the centre.

Cand 2 is a small object about $10^{\prime} \mathrm{SE}$ of M 82 . The direct images indicate a nearly spherical outer LSB component and a small nucleus. The surface brightness profiles are steeper than exponential, as it is common for the faintest dE's (Caldwell \& Bothun 1987). In the $R$ image, a faint arc south of the nucleus is indicated which may be an artefact.

Cand 3 is classified as an Irregular, mainly due to its extended, non-concentric core structure. With $B-R=$ 0.2 , this galaxy is exceptionally blue for M 81 dwarfs $(\langle B-R\rangle=1.23 \pm 0.35$ for the objects studied by BBP98). Note, however, that we have not found evidence for $\mathrm{H} \alpha$ emission. Thus, cand 3 may be a perturbed background galaxy. This interpretation is supported by the fact that two other, slightly perturbed spiral galaxies are located in the vicinity of cand 3 . 

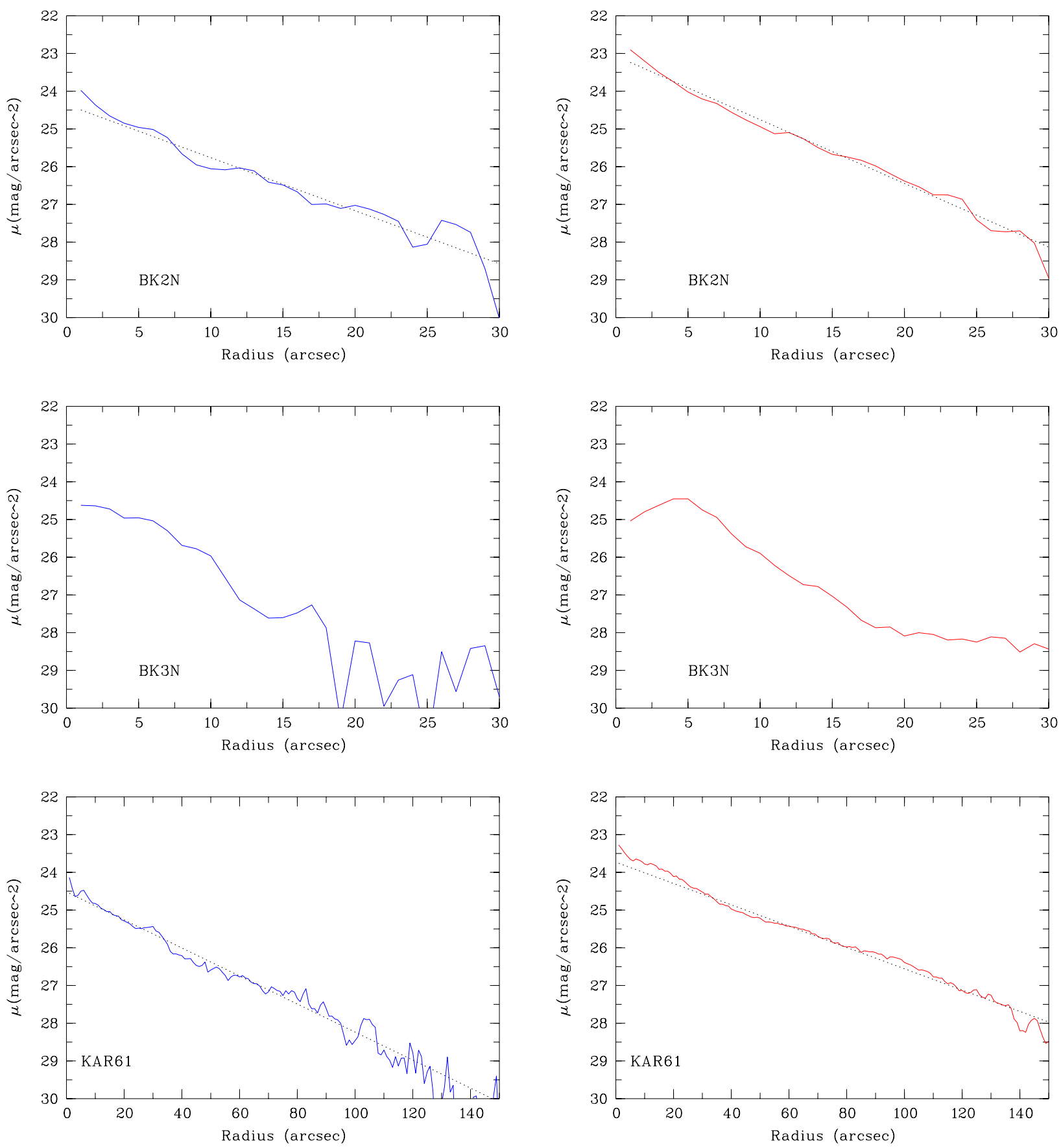

Fig. 10. Surface brightness profiles in $B$ (left) and $R$ (right) for the dwarf galaxies BK $2 \mathrm{~N}$, BK $3 \mathrm{~N}$ and KAR 61 . The dotted lines represent the exponential fits

Cand 4 is an irregularly shaped object located in the outskirts of the peculiar Sc galaxy NGC2976. Thus, it is tempting to speculate that cand 4 may be a satellite of NGC 2976, and that the irregularity is due to tidal distortion from this larger galaxy.
Cand 5 is similar to cand 2, except for the more irregular structure. The profile is well fitted by the exponential model.

Cand 6 is the smallest, and therefore the faintest, of the objects in Table 1 . With respect to size, this object is close to the limit of the survey. The $B$ surface brightness profile is perfectly fitted by an exponential. 

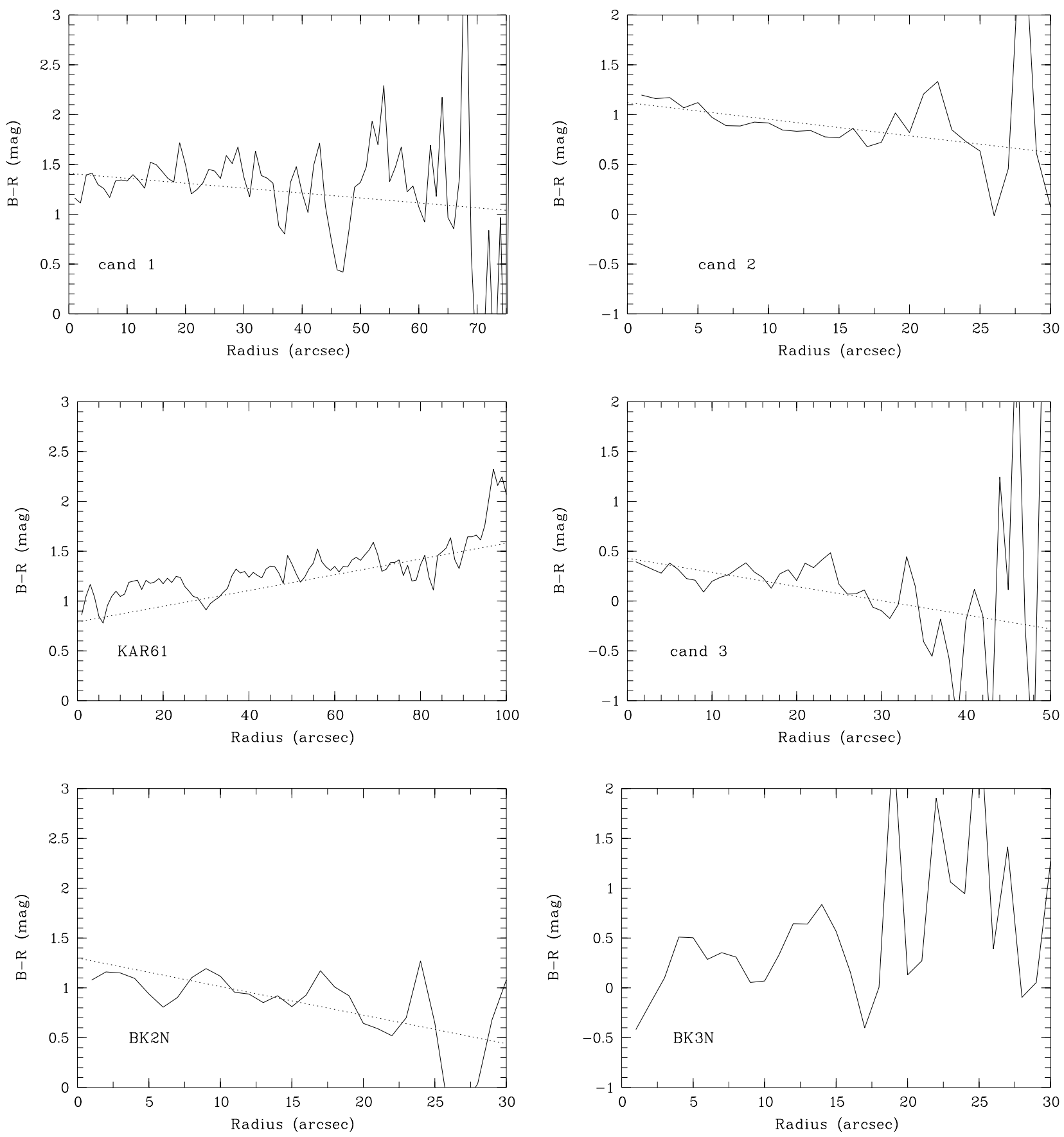

Fig. 11. $(B-R)$ colour profiles of the dwarf galaxy candidates $1,2,3$ and of the dwarf galaxies KAR 61 , BK $2 \mathrm{~N}$ and BK $3 \mathrm{~N}$. The dotted lines result from the exponential fits of the $B$ and $R$ images

\section{Conclusion}

We performed a search for LSB dwarf galaxies in the centre of the M 81 group on digitally stacked Schmidt plates. Based on simulations, we estimate a detection rate of about 80 to 90 per cent for $\mu_{B}(0)<26 \mathrm{mag} / \square^{\prime \prime}$. At fainter magnitudes, the search is strongly affected by the irreg- ularly distributed "Galactic cirrus". Most reliable search results were obtained by a combination of the automated search and a visual inspection.

We found six new candidates for LSB dwarf galaxies in the M81 group. At least one candidate is very likely a group member. This galaxy resembles the dwarf spheroidal BK $5 \mathrm{~N}$, which is the object with the lowest 
central surface brightness among the galaxies studied by BBP98 and is the object with the second-lowest surface brightness among the known M81 group dwarfs. The low central surface brightness in combination with the large scale-length of the newly found object make it an interesting candidate for dwarf galaxies with properties intermediate between $\mathrm{BK} 5 \mathrm{~N}$, which is comparable with normal dwarf spheroidals from the Local Group, and the exceptional LSB dwarf galaxy F8D1 described by Caldwell et al. (1998). Of course, it cannot be unambiguously decided from imaging alone that small LSB objects found in the field around M 81 are really dwarf members of the group. Some candidates may prove as background galaxies. In this case, however, these object are interesting candidates for the rare class of LSB giant galaxies of the type detected by Bothun et al. (1987).

Acknowledgements. The authors wish to thank Jens Brunzendorf for many helpful discussions and suggestions in the context of plate scanning and the reduction of the digitised plates. We acknowledge further René Krieg for his contributions to the image classification software and Uwe Laux for his assistance in surveying the field by eyes. We are also grateful to Freimut Börngen for explaining the strategy of his search for dwarf galaxies on Tautenburg Schmidt plates. Albert Bruch and Renko Ungruhe are acknowledged for providing the object detection software from the Münster Redshift Project. This research has been made use of the NASA/IPAC Extragalactic Database (NED) which is operated by the Jet Propulsion Laboratory, California Institute of Technology, under contract with the National Aeronautics and Space Administration.

\section{References}

Appleton P.N., Siqueira P.R., Basart J.P., 1993, AJ 106, 1664 Binggeli B., Cameron L.M., 1993, A\&AS 98, 297

Börngen F., Karachentseva V.E., 1982, Astron. Nachr. 303, 189

Börngen F., Karachentseva V.E., Schmidt R., Richter G.M., Thänert, W., 1982, Astron. Nachr. 303, 287

Börngen F., Karachentseva V.E., Karachentsev I.D., 1984, Astron. Nachr. 305, 53

Bothun G.D., Impey C.D., Malin D.F., Mould J.R., 1987, AJ 93, 29

Bremnes T., Binggeli B., Prugniel P., 1998, A\&AS 129, 313 (BBP98)
Brunzendorf J., Meusinger H., 1999a, A\&AS 139, 141

Brunzendorf J., Meusinger H., 1999b, in: Treasure-Hunting in Astronomical Plate Archives, Kroll P. et al. (eds.). H. Deutsch, p. 55

Caldwell N., Bothun G.D., 1987, AJ 94, 1126

Caldwell N., Armandroff T.E., Da Costa G.S., Seitzer P., 1998, AJ 115,535

de Vaucouleurs G. 1975, in: Galaxies and the Universe, Sandage A., Sandage M., Kristian J. (eds.). University of Chicago Press, Chicago, p. 557

Hawkins M.R.S., 1991, Working Group of Wide-Field-Imaging, Newsletter 1, 23

Horstmann H., Schücker P., Seitter W., et al., 1989, Bull. Inf. CDS 37, 43

Huchtmeier W.K., Skillman E.D., 1998, A\&AS 127, 269

Irvin J.J., Davis I., Disney M.J., Phillipps S., 1989, MNRAS 245,289

Karachentseva V.E., Schmidt R., Richter G.N., 1984, Astron. Nachr. 305, 59

Karachentseva V.E., Karachentsev I.D., Börngen F., 1985, A\&AS 60, 213

Karachentseva V.E., Karachentsev I.D., 1998, A\&AS 127, 409

Kemp S.N., Meaburn J., 1995, in: The future Utilisation of Schmidt Telescopes, Chapman J.M. et al. (eds.), ASP Conf. Ser. 84, 200

Knox R.A., Hambly N.C., Hawkins M.R.S., MacGillivray H.T., 1998, MNRAS 297, 839

Lehmann H., Häupl W., 1987, in: Astrophotography, Marx S. (ed.). Springer, Berlin, p. 64

Maddox S.J., Sutherland W.J., Efstathiou G., Loveday J., 1990, MNRAS 243, 692

Madore B.F., Freedman W.L., Lee M.G., 1993, AJ 106, 2243

Malin D.F., Carter D., 1993, ApJ 274, 534

Mateo M., 1998, ARA\&A 36, 435

Meusinger H., Brunzendorf J., Froebrich D., Krieg R., 1999, in: Treasure-Hunting in Astronomical Plate Archives, Kroll P. et al. (eds.). H. Deutsch, p. 223

Miller B.W., Hodge P., 1994, ApJ 427, 656

Richter G.M., 1978, Astron. Nachr. 299, 331

Sandage A., 1976, AJ 81, 954

Schweizer F., Seitzer P., 1988, ApJ 328, 88

Tully R.B., 1987, ApJ 321, 280

van Driel W., Kraan-Korteweg R.C., Binggeli B., Huchtmeier W.K., 1998, A\&AS 127, 397

Zickgraf F.J., Humphreys R.M., Sitko M.L., Manley T., 1990, PASP 192, 925 